\title{
Personify Educational Assistance Application for Special Children using Deep Learning
}

\author{
S.Sankara Gomathi, A.Amutha, M.Jayapraksan
}

\begin{abstract}
Despite various Stand-alone educational assistance application for normal children but for the special children it was an exceptional case still, so this children with (Anxiety Disorder, ADHD, Learning Disabilities) find difficult to learn for long hours without getting distracted. A caretaker is needs to be with them at all the time in order to engage them in studying efficiently. Using this technology at its best, Deep Learning can be used to monitor the children when they are distracted and their attention can be drawn back by imposing volunteer distractions on the screen based on the concept of Face Recognition (in terms of facial expressions). The work has been implemented using python \& OpenCV platform. By using this, The scanned image i.e. testing dataset is being compared to training dataset and thus emotion is predicted for incorporating with assisting component.
\end{abstract}

Keywords- Deep Learning, ADHD, Face Recognition, OpenCV

\section{INTRODUCTION}

Deep Learning is one of the subdivisions of machine learning in Artificial Intelligence that has networks, which are capable of learning data representations, rather than any other specific algorithms. This kind of algorithm will completely analyze the structure and function of the brain, in order to solve a host of complex problems using computer that couldn't otherwise be tackled.

Inrecent days, DNN classifiers plays a major role in facial expression classification and to extract the users most representative features with the help ofthe emotion they are exhibit [1].

ADHD (Attention Deficit Hyperactivity Disorder) is the most common mental health disorder in children with the symptoms of hyperactivity, impulsivity and anxiety. About $7 \%$ of children worldwide have this disorder and it becomes a causative one for the health of young generation. The children suffering from ADHD has anxiety disorders, disruptive behavior disorders and higher prevalence of mood disorders. These Symptoms affect the children's overall functioning. Taking the academic perseverance as a major cause and application was developed for the individual use with the knowledge of motivational incentives which trials out the periodic assessment of children with and without the presence of caretakers on their daily needs [2].

Revised Manuscript Received on April 12, 2019.

S.Sankara Gomathi, Department of ECE, Veltech Multitech Dr.Rangarajan Dr.Sakunthala Engineering College, Chennai. T.N ,India. (E-mail: sankaragomathi@veltechmultitech.org)

A.Amutha, Assistant Professor, Ramanujan Centre for higher Mathematics, Alagappa University, Karaikudi, T.N ,India. (E-mail: amutha.angappan@rediffmail.com)

M.Jayapraksan, Joint Director, Directorate General of Training ,MSDE,Govt. of India, New Delhi, India. (E-mail: m.jayaprakasan@gov.in)

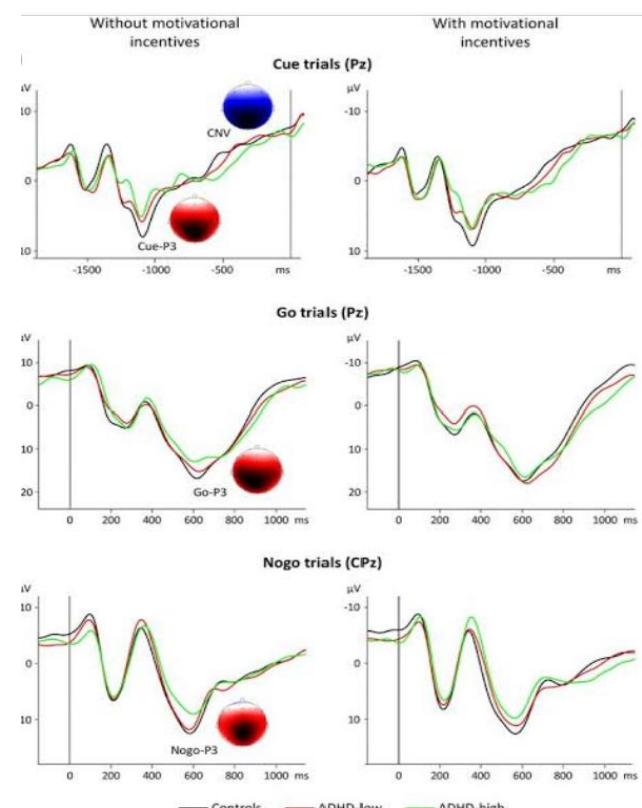

Figure 1: Motivational Acknowledgement

Facial expression classification is a key factor on detecting ones apparent behavior (appearance) towards the systematically undergoing process. In that case appearance based methods are using feature extraction, feature selection and classification methods in order to determine expression on the face [1].

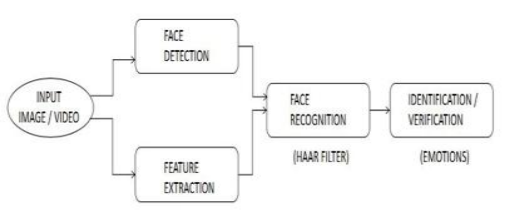

Figure 2: Face Expression Recognition

"Understanding human emotion is an important area of research". In general, one of the reason we choose to focus on the area of facial expression is because of the emotions that has been involved is documented for several years. And the database containing face emotions are being used by the current researchers in order to investigate on facial expression in computer vision named kaggle's facial expression recognition challenges with $71.2 \%$ of accuracy $[4,5]$. 


\section{RELATED WORK}

The study already presented in the related papers evidenced the possibility in supporting the e-learning process for the students with ADHD using aTenDerAH a videogame support to the students on learning [6]. And also the children trained with the help of brainwave changes and neuro feedback based attention [7]. In similar case scenario there also exists an "Augmented Learning Environment" for the children having ADHD. It helps out the teachers as well as students on teaching and learning the concepts. It is mainly helpful and has been proven resolution while reading and spelling problems like dyslexia, in addition with improvements over language, comprehension and memory [8]. Though it is endured with several categories of learning but in some way it is effectuate only based on their attention level namely selective attention, sustained attention, \& divided attention. The existing work has been designed with the greater disadvantage that it resolves out only the compatibility over learning and not the flexibility and involvement towards learning by providing a time gap when they are not interested.

\section{EAA}

The study presented in this paper overcomes the drawback retained in the existing work. As the existing work were fully dealt with only learning by encapsulating the platform with videogame support not figuring out the mind state and involvement of the children. But the work that has been presented in this paper consists of facial expression classification along with the feature extraction on detecting the emotion of the children during learning process. The detected emotion has undergone the stage of classification parameters and it compared with the training parameters for accounting children distraction while learning. If the stage pursue with the condition of distraction then there will be some volunteer distractions on the screen.

\section{METHODOLOGY}

\section{A. SYSTEM ARCHITECTURE}

The complete system architecture is as shown in figure 3 and its algorithm is divided into major two techniques as training and classification. Basically we need to train the networks to classify the emotions of a given face, once the user has started watching the educational video then the face of the user is captured through the webcam and recognition is carried out along with extracted features and compared with the training dataset to know whether the user is distracted or not. If the state terminates with a condition of distraction then the educational video resumes for a few second and the attention gaining video starts playing for a while then continued by the same educational video what the user have already seen. Similarly if their perceives no distraction from the user on seeing educational video, the condition terminates on continue playing the video without any interference. Thus the children can learn efficiently with this application and there is no need for caretakers to take care of them while studying.

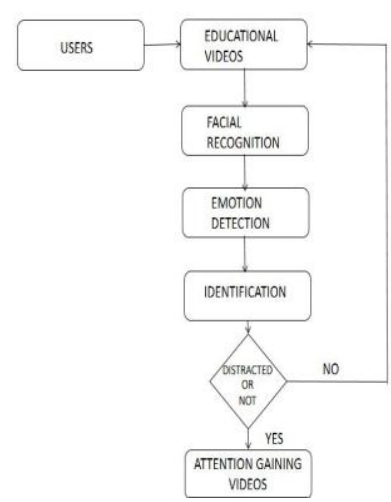

Figure 3: Complete System Workflow

\section{B. IMAGE PROCESSING}

In this concept in order to enhance an image, the particular image is converted to grayscale when the face recognition/feature extraction on processing. The image conversion is done with the methods namely average method \& weighted or luminosity method incorporating the colors as follows [9].

\section{AVERAGE METHOD}

$$
\text { Grayscale }=(\mathrm{R}+\mathrm{G}+\mathrm{B}) / 3
$$

\section{WEIGHTED OR LUMINOSITY METHOD}

$$
\text { Grayscale }=((0.3 * \mathrm{R})+(0.59 * \mathrm{G})+(0.11 * \mathrm{~B}))
$$

Thus the method also involves scanning the image through which the particular information has been extracted for processing the acquired raw image. Once the scanning process is done the processed image will undergo a digitalized enhancement and then the result is obtained which determines the property of image and further classifies the image.

\section{OpenCV (Open Source Computer Vision Library)}

OpenCV is Computer Vision Library, free for all extensive library which consist of more than 2000+ algorithms and parameters categorization used to carry out computer vision and machine learning related projects. The OpenCV formulation involves many tasks such as Face Recognition, Object Identification, Camera Movement Tracking, Scenery Recognition etc $[9,10]$.

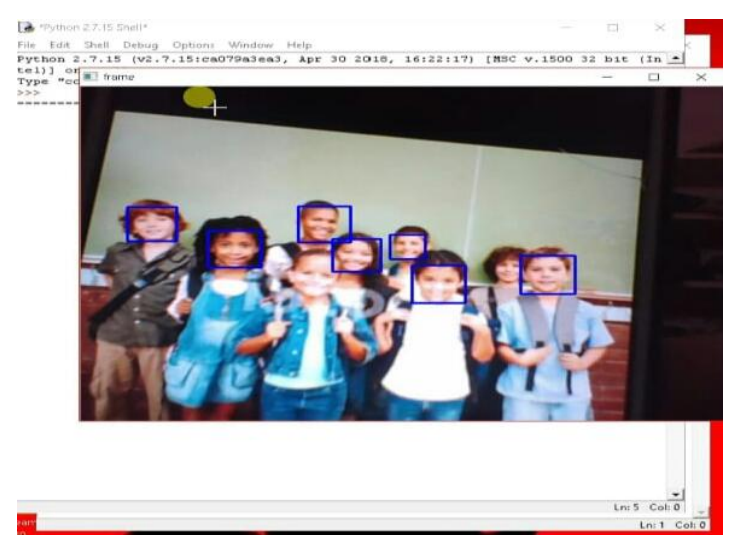

Figure 4: Face Identification 


\section{DATASETS COLLECTION}

With the help of training datasets the detected emotion is compared based on the concept called Principal Component Analysis (PCA). The database that holds the various training parameters are Cohn-Kanade Expression database regarding facial expression and FER2013 datasets labeled with seven basic emotions as follows $[11,12,13,14]$.
a. Neutral condition
b. Happy mood
c. Contempt
d. Anger mind
e. Panic
f. Disgust
g. Surprise
h. Sad

As we seen above Cohn-Kanade Database is one of the databases used for emotion detection. The database holds numerous types of emotions with different categories as first second and third. The First version labeled with "CK" which determines the neutral emotions in accordance to other higher emotions. Similarly, The Secondversion is labeled with "CK+"which increase the frequency of processes and subjects from $25 \%$ to $30 \%$. Whereas the third version will have both the features $\mathrm{CK}$ and $\mathrm{CK}+$ and stillremains in the under development stage[9].

The complete dataset is divided into two categories as shown in the figure namely training and classification parameters where,

- TRAINING PARAMETERS is to teach the classifier to recognize the predicted labels.

- CLASSIFICATION PARAMETERS is to estimate the classifier performance.

Generally PCA is nothing but a statistical procedure to convert a set of observations that were correlated into the linearly correlated variables. In the same way here there exists a set of observations in terms of image acquisition and emotion detection i.e. Source images and Source emotion which were correlated and compared with existing facial emotion database and determine the current mood of the person by Starting with a neutral face and ending with the emotion. Thus the observed emotions were correlated with the existing database [20].

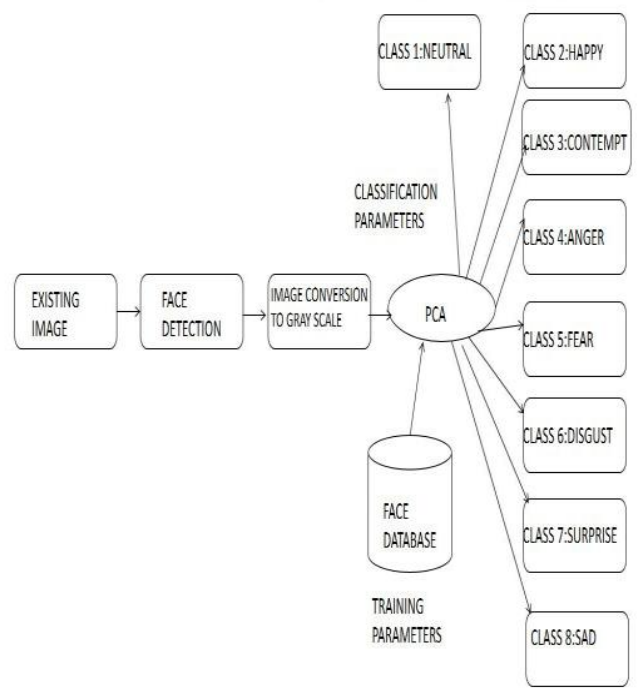

Figure 5: Flow of Emotion Detection
F. STEPS TO PERFORM EMOTION DETECTION USING OPENCV-PYTHON

1. Once the installation is finished, Create the own dataset by analyzing the images in the group or we can use the existing database and the dataset is then organized into two different directories as one contains images and other contains information about the different types of emotions.

2. Run the sample images with the help of python code and the emotions are sorted in the order and perform subsequent encoding.

3. There exist various classes to used under OpenCV for emotion detection with the help of Tensor Flow, but we mainly deal with the Fisher Face one where the emotions are classified based on the Cohn-Kanade AU-coded expression $(\mathrm{CK}+)$ databaseand FER which consist of some basic emotions as "Facial expressions and emotions are continuous entities with numerous variations"[13].

4. Extraction of faces is done through the training and classification set on estimating the performance of images that were extracted and exhibiting the property called size along with feature characterizationusingHAAR cascade classifier [14, $15,19]$.

5. The image is finally converted into grayscale and compilation is done with $80 \%$ of training set and $20 \%$ of classification set [9].

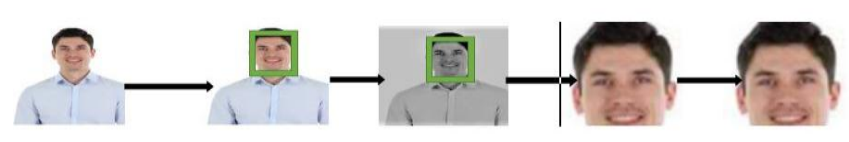

Figure 6: Image Mapping from Color to Grayscale

After the completion of this process the emotion detection mechanism which was coded then interfaced with the user collaborative educational assistance platform designed using python (QT Designer), PyQt is a GUI widgets toolkit. It is a python interface for Qt. A platform that supports GUI library which helps in creating graphical applications. In other words it is a blend of python programming language and Qt library that holds simple drag \& drop interface without writing the code as well as designing the interface by writing the code too [10].

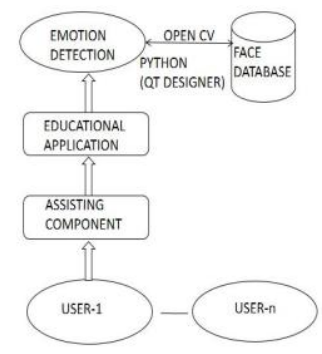

Figure 7: Platform Incorporation while Detecting Emotion 


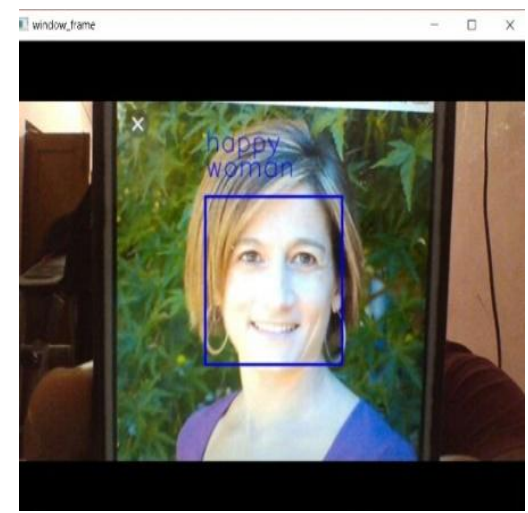

Figure 7.a - HAPPY

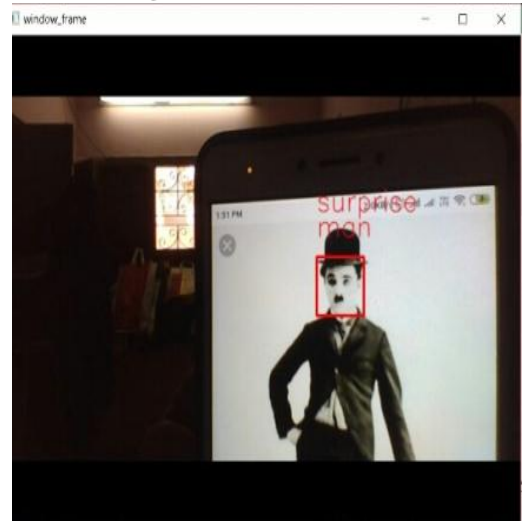

Figure 7.b - SURPRISE

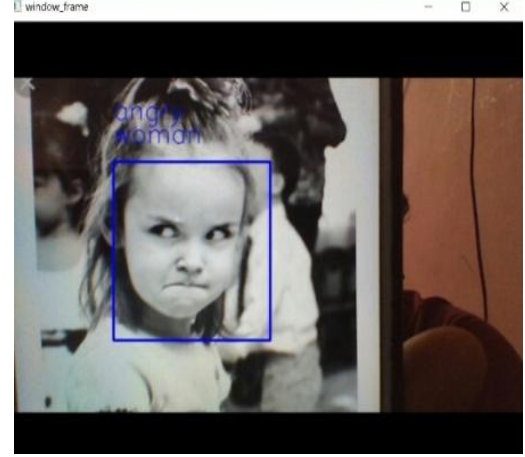

Figure 7.c - ANGRY

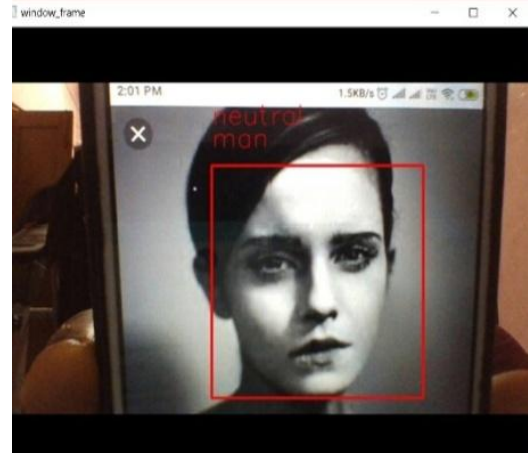

Figure 7.d - NEUTRAL

\section{RESULTS \& EVALUATION}

We used deep learning algorithm for recognizing facial expression and we use a webcam for recognizing face and facial expressions in terms of emotions. The proposed deep network was trained using FER2013 database. The total number of images in database is $35,000+$ and which is compared with the emotion we detected and results whether the person is distracted or not while learning and the volunteer distractions are imposed on the screen when the condition is stated as "Distracted". Since, the emotion detection remains an inseparable part of computer vision with various applications namely improvisation in web development, learning practices, immersive gaming, app and product development. Therefore the implementation is resulted with emotion detection as a major factor in "Educational Assistance Application".

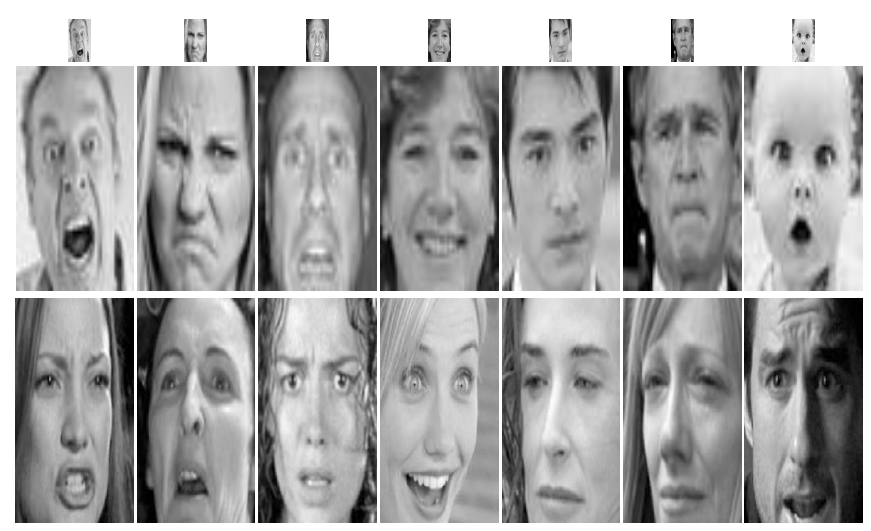

Figure 8: Comparison of FER \& Detected Emotions

\section{CHALLENGES}

The main aim is to develop an educational assistance application based on the emotion detected from a face that was recognized through a webcam. The most challenging part in developing this application is detecting the emotion of the user who use this application because two emotions look quite similar ,i.e. "Disgust" being classified as "Sadness" and "Surprise" being classified as "Happy" and so on for the different categories ofemotions.(Happy, Neutral, Disgust, Sad, Surprise, Anger, Fear, Contempt).

The result obtained for similar case scenario with different categories of emotions provides the $70 \%$ of accuracy (approx) which is quite well what the classifier has learned a bit on detection. So it is important to note that, how we must increase its efficiency as well as accuracy. Similarly, if we look at the emotions list which has only limited number of examples for "Sad", "Fear", "Contempt" [9].

By increasing the images in number for these emotions it is very easy to increase the optimization as well as the increase in optimization can also reach some peak level if we no longer take these emotions into consideration.

\section{CONCLUSION AND FUTURE WORK}

We can very well read the facial expressions using the channel of Non-Verbal Communication. When we think of body language, typically some of the first things that come to our minds are physical motions, hand gestures, and posture. However, one of the most important and easy-toread forms of body language are our facial expressions. Hence we can understand the person emotion on analyzing the emotion in the various stages of recognition. On doing such analyze the person current feeling towards their work is detected (in terms of emotion) and redirected to an entertainment part with this assisting application.

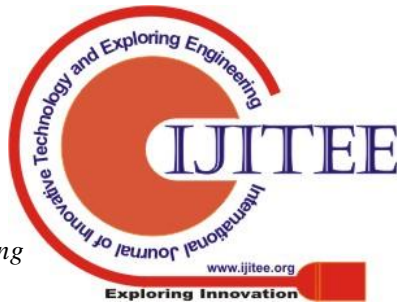


By seeing the tremendous growth of e-learning across worldwide, there exists various learning environment that provides reading and spelling abilities for the children with this disorder. In that case Digitalized solution plays a vital role in current scenario. Though there exists a various digitalized learning environment but some stands out with a lot of drawbacks on involving children in the learning practices as mentioned above. Correspondingly, all the contents are played based on the user's profile. Subsequently, adding intricate details about the human emotions can provide much more pronounced behavioral models of different types of users. The emotions were deployed into two categories as positive and negative estimate. In that case usually positive estimate will not give rise to uncertain conditions but the negative estimate will definitely results in the uncertain conditions and also fosters analytical thinking in order to carry out the critical tasks. Various evidences formulates that emotional state vouches for the better learning practices.

In that point of view the created application works in a similar way of detecting emotion on continuous learning through videos. If the detected emotion terminates the condition of state of distraction the educational video resumes for few second and attention gaining video starts playing for few more seconds along with the time spent for learning .The attention gaining part personified as imposing volunteer distractions on the screen in the state of distraction. Meanwhile if they are not distracted the educational video continues playing without any interference while learning the concepts through videos as shown in Figure.3.

Thus we expect that our proposed application based on emotion detection is being used for many children with "Hyperactivity" disorders and engross the children passion towards learning without any distraction.

\section{REFERENCES}

1. Shabab Bazrafkan, Tudor Nedelcu, Pawel Filipczuk, Peter Corcoran, "Deep Learning for Fcial Expression Recognition: A step closer to a smart phone that knows your moods",ICCE, 2017IEEE.

2. Jarenseri Mitrapanot, Bawanpak Bousai, “iCare-ADHD:A Mobile Application Prototype, ICT-ISPC, 2018.

3. https://www.healthline.com

4. Alexandra Savoiu, JamesWong, "Recognizing Facial Expression Using Deep Learning"-Standford University.

5. https://www.researchgate.net/publication/287867911_Emotio n_Recognition_and_Its_Applications

6. Laura Mancera, Silvia Baldiris, Ramon Tabregat, Sergio Gomez, Carolima Mejia, "aTenDerAH: a VideoGame To Support E-Learning Students With ADHD”, ICALT, 2017 IEEE.

7. Ching-LingChen, Yung-Wen Tang, Nian-Qiaozlang, Jungpil SHIN ,"Neurofeedback Based Attention Training for ChindrenWith ADHD “,2017 IEEE.

8. Eleni Mangina, Giussepe Chiazzese, Tomonori Hasegawa"AHA: ADHD Augmented Learning Environment”,2018 IEEE,NSW,Australia.

9. "Emotion Detection Using Image Processing in Python", Bharathi Vidyapeethi Institute of Computer Application and Management (BVICAM) ,NewDelhi,2018 IEEE.

10. https://OpenCV.Org/ \& https://pythonprogramming.net

11. Ali Mollahosseini, David Chan, and Mohammad H. Mahoor, "Going Deeper in Facial Expression Recognition Using Deep Neural Networks", University of Denver, CO.
12. Hong-Wei Ng, Viet Dung Ngyuen, Vassilios Vonikakkis, Stefan Winkler, "Deep Learning for Emotion Recognition on Small Datasets Using Treansfer Learning", ADSC.

13. - Kanade, T., Cohn, J. F., \& Tian, Y. (2000). Comprehensive database for facial expression analysis. Proceedings of the Fourth IEEE International Conference on Automatic Face and Gesture Recognition (FG'00), Grenoble, France 46-53. Lucey, P., Cohn, J. F., Kanade, T., Saragih, J., Ambadar, Z., \& Matthews, I. (2010). The Extended Cohn-Kanade Dataset $(\mathrm{CK}+)$ : A complete expression dataset for action unit and emotion-specified expression. Proceedings of the Third International Workshop on CVPR for Human Communicative Behavior Analysis (CVPR4HB 2010), San Francisco, USA,94-101

14. https://www.asmag.com/mobile/article_detail.aspx?aid=23883

15. Justus Schwan, Esam Ghaleb, Enrique Hortal and Stylianos Asteriadis, "High Performance and Light Weight Real-Time Deep Face Emotion Recognition",2017 IEEE.

16. Heechul Jung, Sihaeng Lee, Sunjeong Park, Injae Lee, "Development of Deep Learning-Based Facial Expression Recognition System”, ICT R\&D Preogram,2014.

17. Rajesh Kumar G.A, Ravi Kant Kumar, Gowtam Sanyal, "Facial Emotion Analysis Using Deep Convolution Neural Network", ICSPC,Duragpur,2017.

18. Injae Lee, Heechul Jung, Chung Hyuu, Jeongil Seo, Junmo Kim, "Real-Time Personalized Facial Expression Recognition System Based on Deep Learning", ICCE.2016 IEEE.

19. Wathsala Nayomi Widanagmaachchi,"Facial Emotion Recognition With a Neural Network Approach",UCSC, THESIS-Colombo School of Engineering,2009.

20. "Analysis of Emotionally Salient Aspects of Fundamental Frequency for Emotion Detection", Caios Busso,2008 IEEE. 